\title{
Timing of surgery and radiotherapy in the management of metastatic spine disease: A systematic review
}

\author{
EYAL ITSHAYEK ${ }^{1}$, JOSH YAMADA ${ }^{2}$, MARK BILSKY ${ }^{3}$, MEIC SCHMIDT ${ }^{4}$, \\ CHRISTOPHER SHAFFREY ${ }^{5}$, PETER GERSZTEN ${ }^{6}$, DAVID POLLY $^{7}$, ZIYA GOKASLAN $^{8}$, \\ PETER PAUL VARGA ${ }^{9}$ and CHARLES G. FISHER ${ }^{10}$
}

\begin{abstract}
${ }^{1}$ Department of Neurosurgery, Hadassah University Hospital, Jerusalem, Israel; Departments of ${ }^{2}$ Radiation Oncology, and ${ }^{3}$ Neurosurgery, Memorial Sloan-Kettering, New York, NY; ${ }^{4}$ Department of Neurosurgery, University of Utah, Salt Lake City, UT; ${ }^{5}$ Department of Neurosurgery, University of Virginia, Charlottesville, VA; ${ }^{6}$ Department of Neurosurgery, University of Pittsburgh, Pittsburgh, PA; ${ }^{7}$ Department of Orthopaedics, University of Minnesota, Minneapolis, MN; ${ }^{8}$ Department of Neurosurgery, Johns Hopkins University, Baltimore, MD, USA; ${ }^{9}$ Department of Orthopaedics, National Center of Spinal Disorders, Budapest, Hungary; ${ }^{10}$ Division of Spine, Department of Orthopaedics, University of British Columbia, and the Combined Neurosurgical and Orthopaedic Spine Program at Vancouver Coastal Health, Vancouver, BC, Canada
\end{abstract}

Received August 28, 2009; Accepted October 20, 2009

DOI: 10.3892/ijo_00000527

\begin{abstract}
The last decade has witnessed a dramatic change in management of metastatic spine disease, with an increased role for surgery and emerging use of stereotactic radiotherapy, often in combination. Patients may be treated with radiotherapy followed by surgery, or have surgery and then adjuvant radiotherapy. In both cases, the surgeon and oncologist need to select the optimal timing for surgery and radiotherapy to minimize wound complications while obtaining maximum oncolytic effects. The purpose of this review was to determine the optimal timing of surgery and radiotherapy in patients surgically treated for spinal metastases. A systematic review utilizing Medline, Embase, Paper First, Web of Science, Google Scholar, and the Cochrane Database of Systematic Reviews was performed. References were screened to further identify relevant studies and basic science literature reviewed. A total of 46 reports discussing the timing of surgery after radiotherapy, describing experience in 5836 patients, were identified. Only one retrospective study addressed the research question and suggested that surgery within seven days of radiation increases the rate of postoperative wound complications. Timing of adjuvant radiotherapy following surgery was addressed in 51 reports describing 7090 patients. None of the studies specifically answered the research question. The
\end{abstract}

Correspondence to: Dr Charles Fisher, Blusson Spinal Cord Centre, 6th floor, 818 W. 10th Avenue, Vancouver, BC V5Z 1M9, Canada

E-mail: charles.fisher@vch.ca

Key words: metastatic spine disease, stereotactic radiotherapy, adjuvant radiotherapy time interval between radiotherapy and surgery was reported as 5-21 days in nine studies. Based on this systematic review together with the understanding of general principles of wound healing and effects of radiation on wound healing, the optimal radiotherapy-surgery/surgery-radiotherapy time interval should be at least one week to minimize wound complications.

\section{Contents \\ 1. Introduction \\ 2. Collection of the clinical data \\ 3. Evaluation of the data \\ 4. Discussion and conclusions}

\section{Introduction}

Radiotherapy has traditionally been the mainstay of treatment for metastatic disease of the spine. Surgical indications were limited to neurologic deterioration during radiation and failure of radiation therapy, with a limited recognition of issues related to spinal instability. With advancements in biomaterials, spinal biomechanics, and imaging, along with innovations in surgical techniques, came an appreciation for spinal instability as a valid indication for surgical stabilization. Recently, the understanding that radiotherapy and surgery should often be combined in the management of both primary and metastatic tumors of the spine has reached maturity. This is especially true in the treatment of spine metastases, where several landmark studies demonstrated the benefit and cost effectiveness of surgery plus radiation vs. radiation alone (1-3). There are, however, potential adverse events associated with radiation delivered in close proximity to surgery, especially complications related to wound healing (4-6). 
The approach to metastatic disease of the spine is undergoing a paradigm shift as a result of new technology in both the medical and surgical domains, thus the spine surgeon and radiation oncologist often encounter two different clinical scenarios. In the first scenario, a patient with spinal metastases has been treated with radiotherapy and then presents with mechanical back pain, progression of deformity, or neurological deterioration; surgical treatment for decompression and stabilization is thus indicated. In the second scenario, a patient who has had surgical treatment for metastatic cord compression subsequently requires radiotherapy. In the first scenario, the surgeon and oncologist need to decide the best timing for surgery in relation to previous radiotherapy, and in the second case the timing for radiation in relation to previous surgery is the issue. Although clinicians are asking these questions on a daily basis, the literature fails to provide clear guidelines, and in many cases treatment is given based on personal experience and educated hunches.

The objective of this study was to perform a systematic review of the literature relating to the optimal timing for surgery in previously irradiated patients, and for radiotherapy in previously operated patients, with consideration of wound healing complications. The advantages of a systematic review include transparency in the methodology, allowing readers to determine for themselves the acceptability of the process, and the reduction of bias and random error that are inherent to some case series and narrative reviews. A systematic review can thus be an important tool for reaching conclusions from literature that may prove difficult to interpret on a case-by-case basis.

\section{Collection of the clinical data}

Inclusion criteria. Inclusion criteria included: i) a population of patients with primary malignancy or metastatic disease of the spine and a minimum age of 18 years, and ii) intervention of spine surgery through a posterior approach, preceded or followed by radiotherapy. In studies meeting inclusion criteria, we extracted data regarding postoperative wound complicationwound infection, wound breakdown, and delayed wound healing. Articles not meeting the inclusion criteria were excluded from the review.

Literature search to identify primary studies. A comprehensive literature search was performed to locate relevant potential studies. Electronic database searches of Medline (1966November 2007) and Embase (1980-November 2007) were performed using both medical subject headings $(\mathrm{MeSH})$ and text word searching (Table I); Papers First, Web of Science, Google Scholar, and the Cochrane Database of Systematic Reviews were searched using text words. The major review articles were identified, and their reference lists were manually searched for additional citations. Experts in the field were sought and questioned as to possible additional references.

Study selection. Two independent reviewers (E.I. and C.F.) used a standardized study selection worksheet to evaluate the eligibility of each study. The reviewers were blinded to author, institution, and journal of publication. Articles were excluded based on abstract review if both reviewers independently determined that inclusion criteria were not met. Otherwise, all the
Table I. Search criteria.

\begin{tabular}{ll}
1 & Spine or spinal \\
2 & Radiotherapy or radiation or irradiation \\
3 & Surgery or laminectomy or vertebrectomy \\
4 & $\begin{array}{l}\text { Wound or complication or infection or } \\
\text { breakdown or dehiscence }\end{array}$ \\
5 & Cancer or neoplasm \\
6 & 1 and 2 and 3 and 4 and 5 \\
\hline
\end{tabular}

remaining studies were assessed using the complete reports. Any disagreements between observers were resolved through discussion.

General literature search. Because of the paucity of literature discussing the optimal timing of surgical and radiotherapy procedures in relation to wound healing for patients with primary and secondary spine tumors, we used the general medical literature and the literature on physiology of radiation and wound healing to provide necessary background to better answer the questions. A comprehensive search in the general clinical literature was performed, to identify randomized controlled studies and prospective case series that were designed specifically to answer our research questions in different medical disciplines.

\section{Evaluation of the data}

A total of 55 studies were identified in the electronic search. Fourteen were classified as possible for inclusion, but only five fully satisfied the two inclusion criteria (7-11). An additional 61 studies that met inclusion criteria were identified by a bibliography search.

Timing of surgery after radiotherapy. A total of 46 studies that met inclusion criteria, describing 5836 patients who underwent surgery after radiotherapy were identified (Table II). These studies were either surgical case series including some patients who had preoperative radiotherapy, or series describing patients managed primarily with radiation who needed continued treatment with surgery. Only one retrospective study was designed to address the question of timing of radiotherapy and postoperative complications (7). Six studies mentioned the time interval between radiotherapy and subsequent surgery $(2,7,12-15)$.

Eight studies included analysis of the association between preoperative radiotherapy and postoperative complication rates $(9,14-20)$. The relationship was significant in four studies, but did not reach statistical significance in four others. From 15 studies we were able to extract information on 309 previously irradiated patients that were treated surgically, with wound complication rates of $11-50 \%(2,7,8,10,14,21-30)$.

Only three studies included information on the timing of surgery after radiotherapy, and also provided enough information on wound-related complication rates. Ghogawala et al (7) found that patients who underwent surgery after radiotherapy had a $32 \%$ risk of wound-related complications compared with $12 \%$ for those who underwent surgery as the 


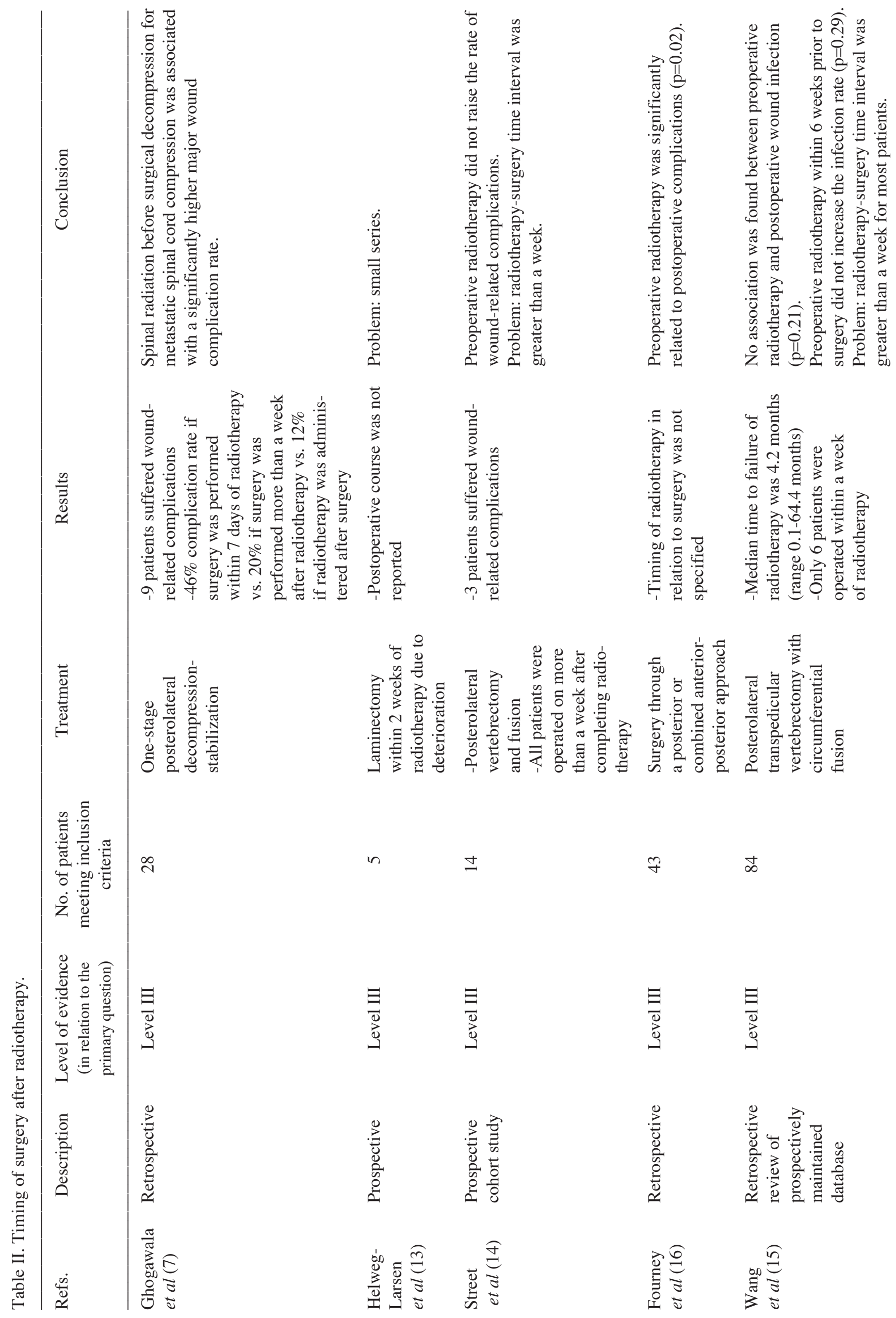




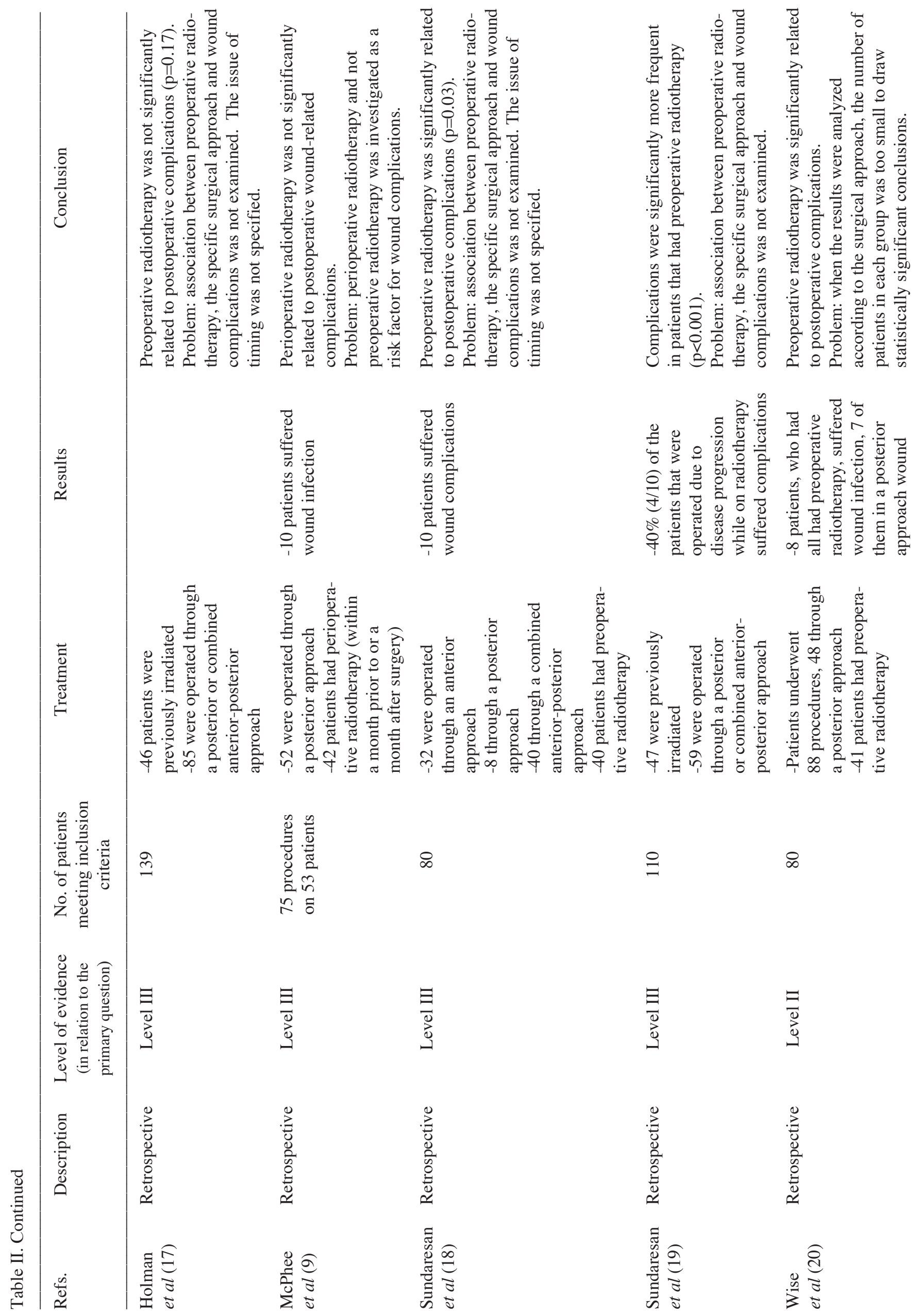




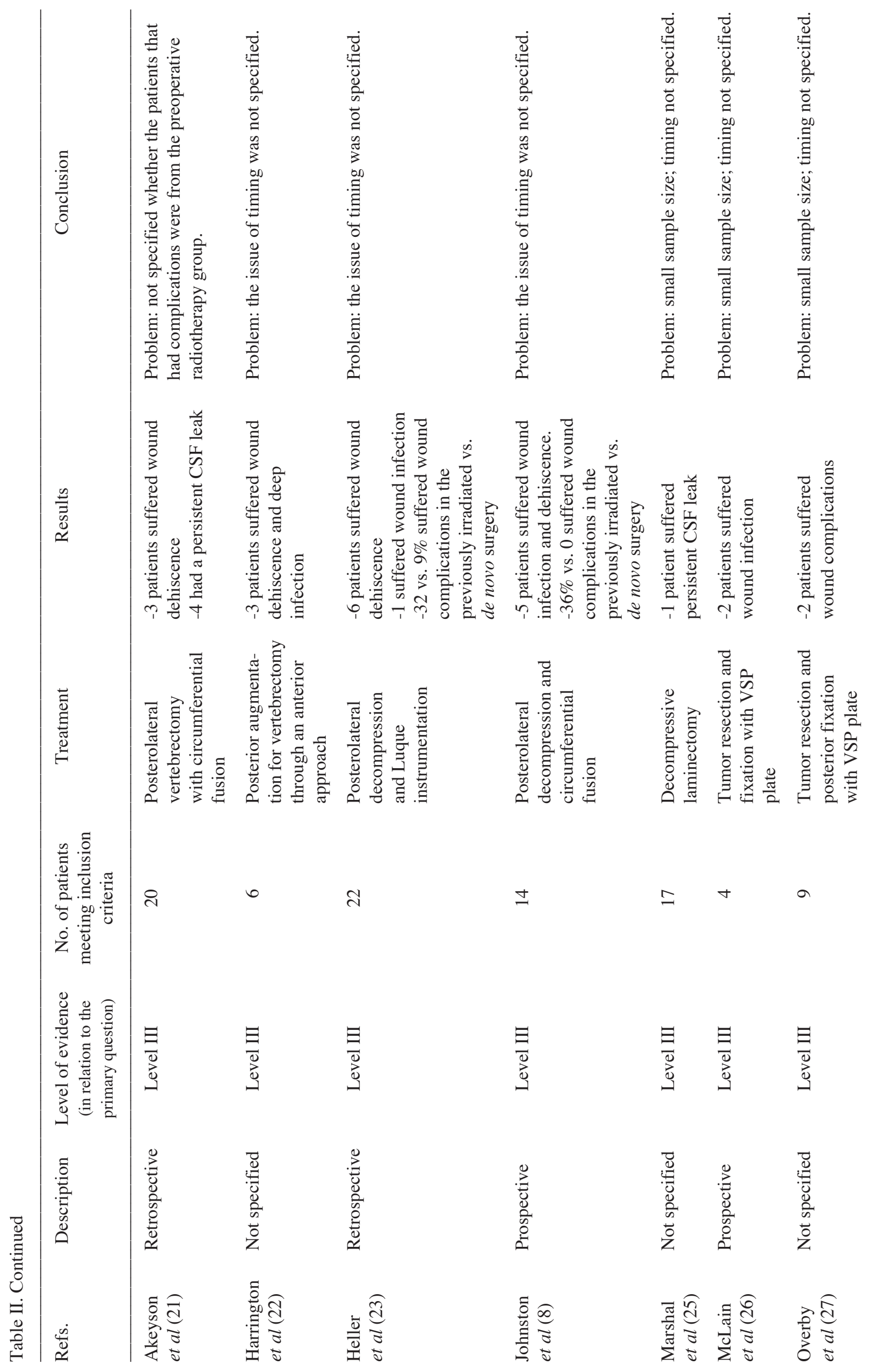




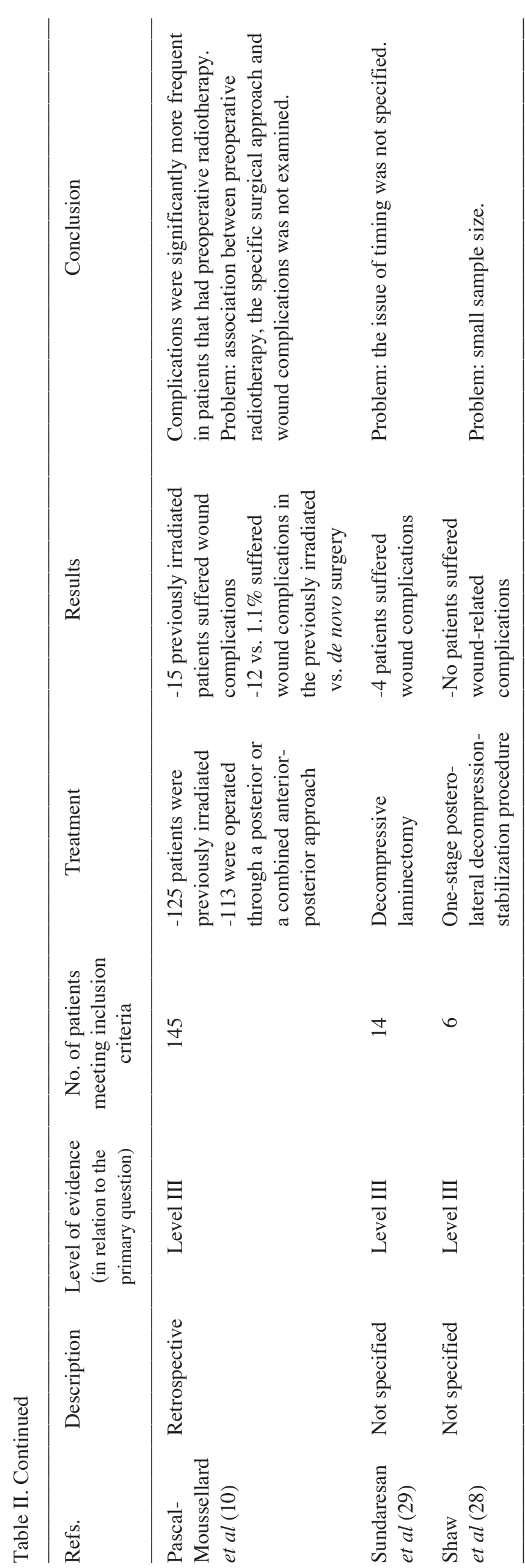

initial therapy. These authors also found that the wound complication rose to $46 \%$ when surgery was performed within seven days of radiotherapy, compared with $20 \%$ if the radiation surgery interval was longer then a week. Patchell et al (2) also showed a 30\% wound-related complication rate in patients who failed radiotherapy and required rapid surgical inter-vention. Most of these surgeries occurred within a week of radiation. Interestingly, Street et al (14) found that approxi-mately $20 \%$ of patients who where treated with posterolateral vertebrectomy, with or without preoperative radiotherapy, experienced major wound-related complications. Surgery was never performed within the first week after radiotherapy in patients in the Street series.

Timing of radiotherapy after surgery. A total of 51 studies that met inclusion criteria, describing 7090 patients who underwent radiotherapy after surgery, were identified (Table III). None of these studies was designed to specifically address the question of timing. To address the question of the timing of radiotherapy following surgery, we extracted information from numerous case series describing, in part, patients who were treated with surgery followed with radiotherapy for metastatic spinal disease. Nine studies reported the surgeryradiation time interval to be 5 to 21 days (2,31-38). One study found no significant association between postoperative radiotherapy and complications (20).

Only eight studies encompassing 122 patients treated with surgery and postoperative radiotherapy presented complete data. Among these, only nine patients $(7.4 \%)$ suffered postoperative wound-related complications. Five studies reported no complications $(7,21,24,28-30,35,38)$. Among these eight, only studies by Young et al (38) and Laohacharoensombat et al (35) reported the surgery-radiotherapy interval, which was 7 and 14 days, respectively.

\section{Discussion and conclusions}

The question of when to operate on or irradiate a patient is encountered frequently in spine oncology. Despite advances in radiation oncology (e.g. radiosurgery) and innovative minimally invasive surgical techniques that have broadened treatment options for this patient population, use of conventional radiotherapy and surgery will continue. In this systematic review, we attempted to establish whether the literature provides information regarding the timing of combined surgery and radiation in a patient with primary or metastatic neoplasia of the spine. Because of the paucity of literature in regards to our research questions, we also reviewed the general medical and basic science literature for additional supporting evidence.

Timing of surgery after radiotherapy. Among the many variables that influence wound healing, two issues appear to have special importance in patients with primary or secondary spine neoplasia who have received preoperative radiotherapy: the total radiation dose and the radiation-surgery time interval (39). In patients with malignant neoplasia of the spinal column, the spinal cord tolerance to radiation determines the maximal radiation dose.

The radiation-surgery time interval was the subject of early studies by Zelman et al (39), who demonstrated in a rat model 


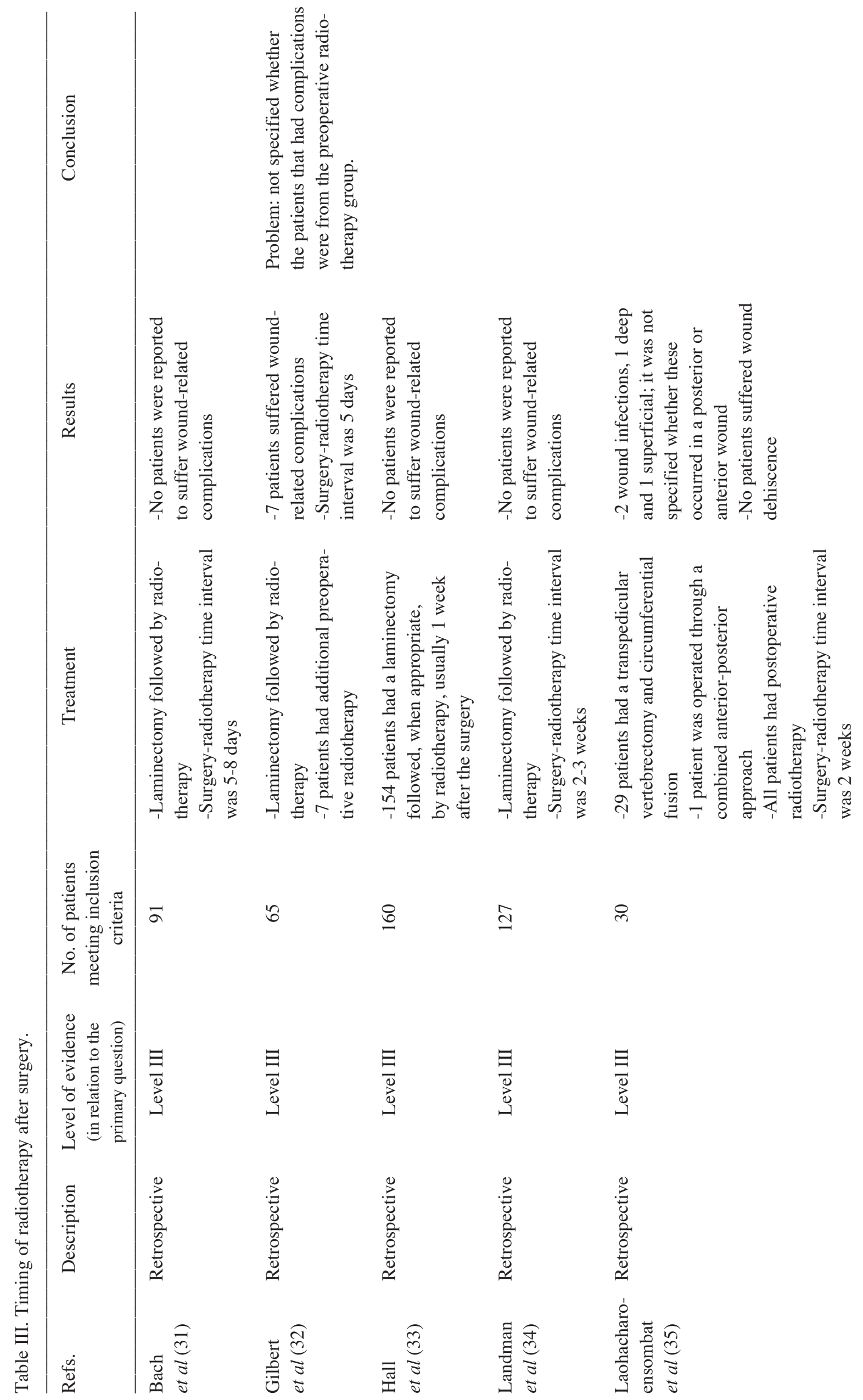




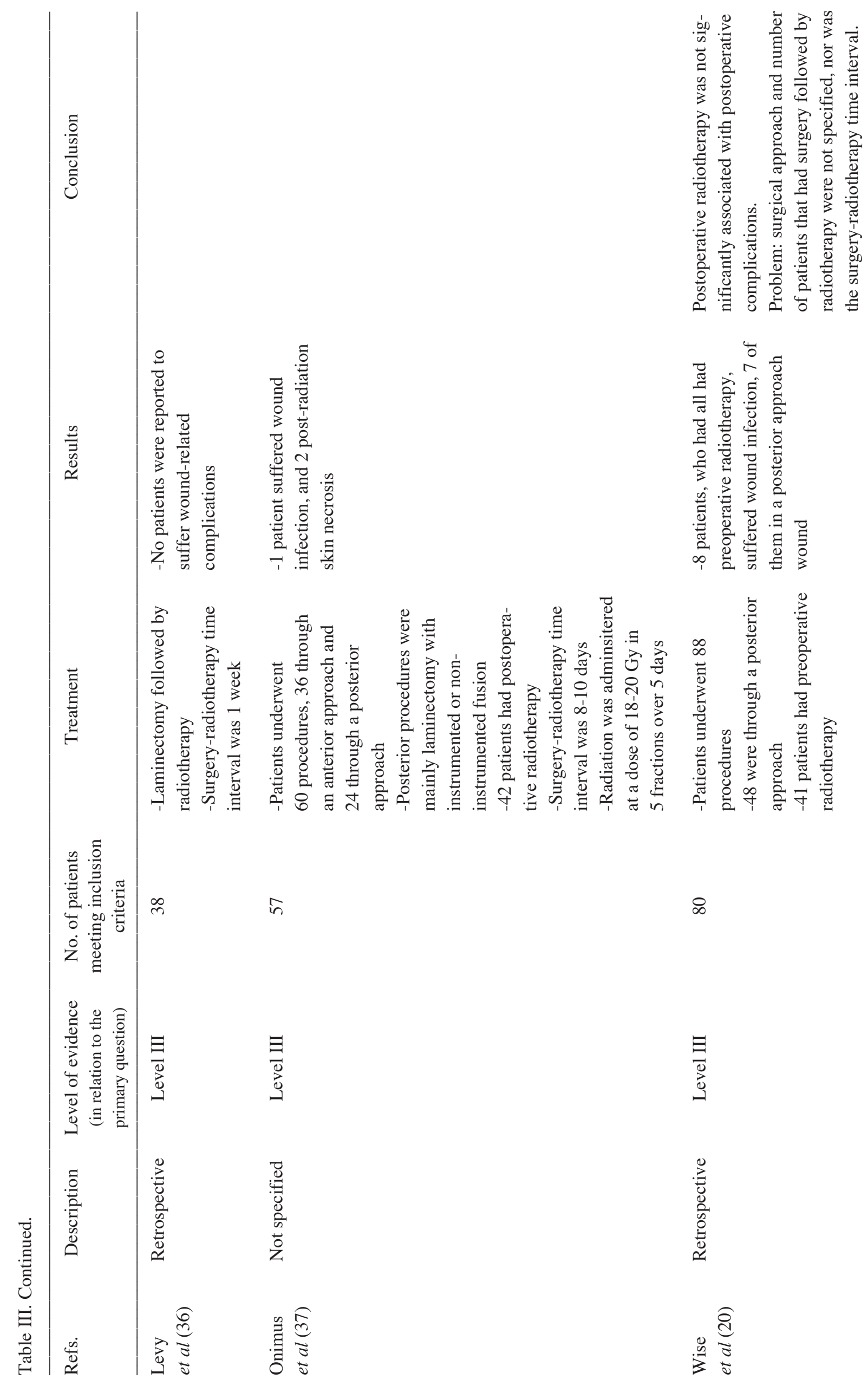




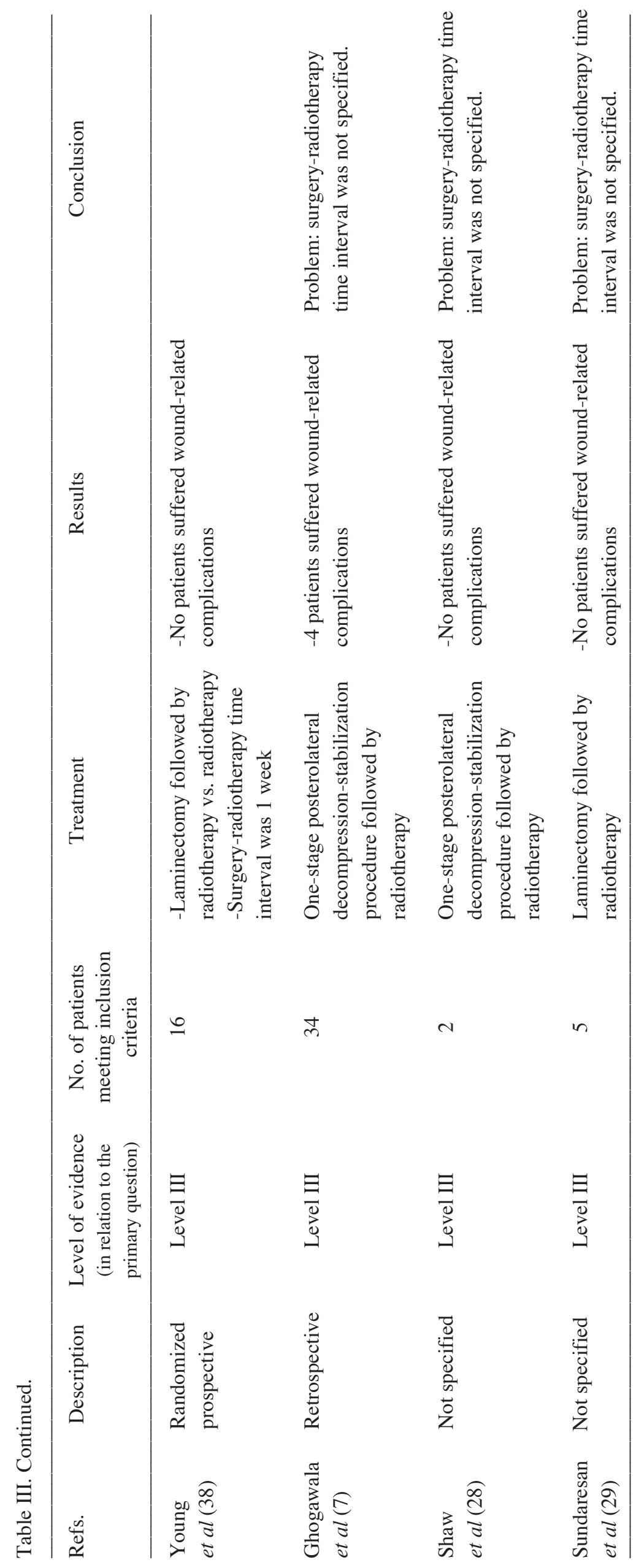


that wound tensile strength decreases when the surgery is done immediately or within one week after irradiation, compared with surgery that is done later. These findings were strengthened by Devereux et al (40) who showed similar results. These authors irradiated rats one week before or on the day of surgery and found the wound-breaking strength decreased by $59 \%$ compared with that of a control group of rats that were not irradiated.

We found only three clinical studies that discussed complications in comparison with the length of time between radiation and surgery. Wang et al (15) retrospectively reviewed a prospectively maintained database comprised of 140 patients who were treated with posterolateral transpedicular decompression for metastatic spine disease. Eighty-four patients had preoperative radiotherapy, of whom only 14 had radiotherapy within the month before surgery. The authors reported a total wound-related complication rate of $11.4 \%$ (16 patients), and found no statistically significant association between preoperative radiation therapy and postoperative infection $(\mathrm{P}=0.21)$. They found no increase in infection rate among patients who had radiation therapy within six weeks prior to surgery $(\mathrm{P}=0.29)$. The authors explained this lack of statistical significance as being due to the fact that only six of their patients underwent surgery within a week after radiotherapy.

Our group has reported outcomes in 42 patients who had posterolateral vertebrectomy for spine metastases (14) including 14 patients $(33 \%)$ who had had preoperative radiotherapy more than one week prior to surgery. Three of the 14 (21\%) had postoperative wound complications, as did seven other patients presented in the studies we reviewed. On the other hand, Ghogawala et al (7) showed a significant association between preoperative radiotherapy and postoperative complication rates in patients with metastatic spinal cord compression. They also reported a $46 \%$ complication rate in 13 patients whose surgery was performed within seven days of radiotherapy vs. a $20 \%$ rate in the 15 patients who had surgery more than a week after irradiation. Although there is a paucity of clinical data, we recommend waiting at least one week after radiation before surgery is undertaken in patients with spinal metastases. The evidence for the recommendation rests on data from a single study (7) and on general principles of the effects of radiation on wound healing from the basic science literature.

Timing of radiotherapy after surgery. Normal wound healing after surgery consists of three stages and requires two years. The mature scar will gain tensile strength equal to $80 \%$ of normal skin. The first week includes the inflammatory stage (0-4 days after injury) and the early phase of the proliferative stage (2-21 days after injury). Stages one and two are critical for wound healing, and very sensitive to radiation (41). These two early stages are characterized by active migration and proliferation of inflammatory cells, and then proliferation of collagen-producing fibroblasts, which have high sensitivity to radiation (42). If radiotherapy is given immediately after surgery, the inflammatory response is inhibited and the number of inflammatory cells is reduced (43). When there is exposure to radiation during the early proliferative phase, when fibroblasts normally proliferate and collagen production approaches its peak, deposition of collagen I and III is inhibited
$(43,44)$. The last stage of healing, granulation, begins five days after surgery. Granulation involves remodeling and maturation of the wound, processes that are less sensitive to radiation (45).

One week after the surgery, the cascade of wound healing has been initiated and collagen production by the fibroblasts has nearly peaked. Devereux et al (40) showed that wound breakdown strength in rats irradiated a week after wounding was similar to that in control rats that had no irradiation. Other research has suggested that the 'safe' surgery-radiation time interval in animals is even shorter, 24-48 h (46). In a parallel study of patients with soft tissue sarcoma, Ormsby et al (47) found that delaying brachytherapy by five to eight days after surgery decreased the wound complication rate from 48 to $14 \%$. Although the directness of this evidence might be questioned because the radiation was not a randomized controlled trial, we felt the evidence was applicable to the primary question of our study and therefore was included.

In an assessment of possible factors associated with higher complication rates in patients undergoing surgery for metastatic spine disease, Wise et al (20) found that postoperative radiotherapy was not a statistically significant risk factor. Among the 51 case series involving patients with spine metastases who were treated with surgery followed by radiotherapy that were systematically reviewed for this study, only eight studies reported wound-related complications, with an average rate of $3.9 \%$ (range $0-16 \%)(7,21,24,28-30,35,37)$. Only two reports detail both the complication rate and the surgeryradiotherapy time interval. Laohacharoensombat et al (35) described 30 patients who had radiotherapy 14 days after the surgery; only one patient $(7.1 \%)$ developed a superficial wound infection. Young et al (37) reported on 16 patients who were given radiotherapy a week postoperatively; none developed wound-related complications.

Although skin that is wounded in surgery becomes a site of proliferation and repair, and remains a weak link until the wound finally reaches maturity approximately two years after the injury, it is the during the early stages of healing, which last about one week, that the risk of wound-related complications seems to be greatest. Irradiating a wound, especially in the first week after surgery, significantly interferes with the normal healing process.

Wound-healing problems are further amplified in oncology patients, who are often malnourished, may suffer from comorbid conditions, and may receive treatment involving corticosteroids, chemotherapy, or antiangiogenesis therapy. In such an unforgiving environment, the clinician has to weigh carefully the desire to control the tumor against the aim of preserving neurological function and maintaining a low complication rate.

Although there is a paucity of clinical data, the best available evidence would suggest avoiding radiotherapy for one week or longer after surgery for spinal metastases through a posterior approach may limit adverse effects on wound healing. The evidence for the recommendation rests on data from a single study involving brachytherapy (47), on the lack of identification of radiation administered at least a week after surgery as an independent risk factor for wound complications in the spine literature, and on general principles of wound healing biology. Although radiation after surgery is not commonly urgent, some tumors such as melanoma will 
benefit from early therapy. Furthermore, logistic and patient scheduling issues also speak to the benefit of having guidelines for the timing of postoperative radiation.

Our study is limited by the small number of studies that discuss the optimal timing of surgical and radiotherapy procedures in relation to wound healing for patients with primary and secondary spine tumors. In addition, a critical appraisal of these studies would provide, at best, Class III evidence, according to the criteria for the classification of evidence developed by the U.S. Preventative Services Task Force (48). However, we believe that it is possible to make preliminary recommendations regarding our two questions. These recommendations provide the best available evidence to date, and may be applied in the context of clinical experience and circumstances, and patient preference pending stronger evidence.

This review provides a foundation on which to move forward with a large prospective multi-center database to generate the numbers and cohorts needed to address these two questions with a higher level of scientific integrity. A further limitation of our review is that the total dose of radiation was not available for analysis in the majority of the reviewed studies. Current radiation treatment planning often includes three-dimensional conformal radiation dosing or stereotactic spinal radiotherapy. These treatment modalities may minimize the radiation dose to the skin at the surgical incision site. These important issues will also require investigation in future studies.

In conclusion, the authors recommend that the radiotherapysurgery time interval should be at least one week for patients with previous radiotherapy. In the opposite scenario, when radiotherapy is given after surgery, a time interval of at least one week should also be maintained.

\section{References}

1. Falicov A, Fisher CG, Sparkes J, et al: Impact of surgical intervention on quality of life in patients with spinal metastases. Spine 31: 2849-2856, 2006

2. Patchell RA, Tibbs PA, Regine WF, et al: Direct decompressive surgical resection in the treatment of spinal cord compression caused by metastatic cancer: a randomised trial. Lancet 366 : 643-648, 2005.

3. Thomas KC, Nosyk B, Fisher CG, et al: Cost-effectiveness of surgery plus radiotherapy versus radiotherapy alone for metastatic epidural spinal cord compression. Int J Radiat Oncol Biol Phys 66: 1212-1218, 2006.

4. Chadwick MA, Vieten D, Pettitt E, et al: Short course preoperative radiotherapy is the single most important risk factor for perineal wound complications after abdominoperineal excision of the rectum. Colorectal Dis 8: 756-761, 2006.

5. O'Sullivan B, Davis AM, Turcotte R, et al: Preoperative versus postoperative radiotherapy in soft-tissue sarcoma of the limbs: a randomised trial. Lancet 359: 2235-2241, 2002.

6. Schwartz SR, Yueh B, Maynard C, et al: Predictors of wound complications after laryngectomy: A study of over 2000 patients. Otolaryngol Head Neck Surg 131: 61-68, 2004.

7. Ghogawala Z, Mansfield FL and Borges LF: Spinal radiation before surgical decompression adversely affects outcomes of surgery for symptomatic metastatic spinal cord compression. Spine 26: 818-824, 2001.

8. Johnston FG, Uttley D and Marsh HT: Synchronous vertebral decompression and posterior stabilization in the treatment of spinal malignancy. Neurosurgery 25: 872-876, 1989.

9. McPhee IB, Williams RP and Swanson CE: Factors influencing wound healing after surgery for metastatic disease of the spine. Spine 23: 726-733, 1998.

10. Pascal-Moussellard H, Broc G, Pointillart V, et al: Complications of vertebral metastasis surgery. Eur Spine J 7: 438-444, 1998.
11. Sundaresan N, DiGiacinto GV, Krol G, et al: Spondylectomy for malignant tumors of the spine. J Clin Oncol 7: 1485-1491, 1989.

12. Bilsky MH, Boland P, Lis E, et al: Single-stage posterolateral transpedicle approach for spondylectomy, epidural decompression, and circumferential fusion of spinal metastases. Spine 25: 2240-2250, 2000.

13. Helweg-Larsen S, Sorensen PS and Kreiner S: Prognostic factors in metastatic spinal cord compression: a prospective study using multivariate analysis of variables influencing survival and gait function in 153 patients. Int J Radiat Oncol Biol Phys 46: 1163-1169, 2000.

14. Street J, Fisher C, Sparkes J, et al: Single-stage posterolateral vertebrectomy for the management of metastatic disease of the thoracic and lumbar spine: a prospective study of an evolving surgical technique. J Spinal Disord Tech 20: 509-520, 2007.

15. Wang JC, Boland P, Mitra N, et al: Single-stage posterolateral transpedicular approach for resection of epidural metastatic spine tumors involving the vertebral body with circumferential reconstruction: results in 140 patients. Invited submission from the Joint Section Meeting on Disorders of the Spine and Peripheral Nerves, March 2004. J Neurosurg Spine 1: 287-298, 2004.

16. Fourney DR, Abi-Said D, Lang FF, et al: Use of pedicle screw fixation in the management of malignant spinal disease: experience in 100 consecutive procedures. J Neurosurg 94: 25-37, 2001.

17. Holman PJ, Suki D, McCutcheon I, et al: Surgical management of metastatic disease of the lumbar spine: experience with 139 patients. J Neurosurg Spine 2: 550-563, 2005.

18. Sundaresan N, Rothman A, Manhart K, et al: Surgery for solitary metastases of the spine: rationale and results of treatment. Spine 27: 1802-1806, 2002

19. Sundaresan N, Sachdev VP, Holland JF, et al: Surgical treatment of spinal cord compression from epidural metastasis. J Clin Oncol 13: 2330-2335, 1995.

20. Wise JJ, Fischgrund JS, Herkowitz HN, et al: Complication, survival rates, and risk factors of surgery for metastatic disease of the spine. Spine 24: 1943-1951, 1999.

21. Akeyson EW and McCutcheon IE: Single-stage posterior vertebrectomy and replacement combined with posterior instrumentation for spinal metastasis. J Neurosurg 85: 211-220, 1996.

22. Harrington KD: Anterior decompression and stabilization of the spine as a treatment for vertebral collapse and spinal cord compression from metastatic malignancy. Clin Orthop Relat Res 233: 177-197, 1988.

23. Heller M, Macnab T and Perrin R: Treatment of metastatic disease of the spine with posterolateral decompression and luque instrumentation. Neuro-Orthopedics 2: 70-74, 1986.

24. Macedo $\mathrm{N}$ and Galicich JH: Decompressive laminectomy for metastatic cancer: What are the current indications? Proc Am Soc Clin Oncol 4: 278, 1985.

25. Marshall LF and Langfitt TW: Combined therapy for metastatic extradural tumors of the spine. Cancer 40: 2067-2070, 1977.

26. McLain RF, Kabins M and Weinstein JN: VSP stabilization of lumbar neoplasms: technical considerations and complications. J Spinal Disord 4: 359-365, 1991.

27. Overby MC and Rothman AS: Anterolateral decompression for metastatic epidural spinal cord tumors. Results of a modified costotransversectomy approach. J Neurosurg 62: 344-348, 1985.

28. Shaw B, Mansfield FL and Borges L: One-stage posterolateral decompression and stabilization for primary and metastatic vertebral tumors in the thoracic and lumbar spine. J Neurosurg 70: 405-410, 1989.

29. Sundaresan N, Galicich JH and Lane JM: Harrington rod stabilization for pathological fractures of the spine. J Neurosurg 60 : 282-286, 1984.

30. Sundaresan N, Scher H, DiGiacinto GV, et al: Surgical treatment of spinal cord compression in kidney cancer. J Clin Oncol 4: 1851-1856, 1986.

31. Bach F, Larsen BH, Rohde K, et al: Metastatic spinal cord compression. Occurrence, symptoms, clinical presentations and prognosis in 398 patients with spinal cord compression. Acta Neurochir (Wien) 107: 37-43, 1990.

32. Gilbert RW, Kim JH and Posner JB: Epidural spinal cord compression from metastatic tumor: diagnosis and treatment. Ann Neurol 3: 40-51, 1978.

33. Hall AJ and Mackay NN: The results of laminectomy for compression of the cord or cauda equina by extradural malignant tumour. J Bone Joint Surg Br 55: 497-505, 1973.

34. Landmann C, Hunig R and Gratzl O: The role of laminectomy in the combined treatment of metastatic spinal cord compression. Int J Radiat Oncol Biol Phys 24: 627-631, 1992. 
35. Laohacharoensombat W and Wajanavisit W: Spinal metastasis: results of surgical management. J Orthop Surg 5: 3-9, 1997.

36. Levy WJ, Latchaw JP Jr, Hardy RW, et al: Encouraging surgical results in walking patients with epidural metastases. Neurosurgery 11: 229-233, 1982.

37. Onimus M, Schraub S, Bertin D, et al: Surgical treatment of vertebral metastasis. Spine 11: 883-891, 1986.

38. Young RF, Post EM and King GA: Treatment of spinal epidural metastases. Randomized prospective comparison of laminectomy and radiotherapy. J Neurosurg 53: 741-788, 1980.

39. Zelman D, Song IC, Porteous DD, et al: The effect of total body irradiation on wound healing and the hematopoietic system in mice. Bull N Y Acad Med 45: 293-300, 1969.

40. Devereux DF, Kent H and Brennan MF: Time dependent effects of Adriamycin and $\mathrm{x}$-ray therapy on wound healing in the rat. Cancer 45: 2805-2810, 1980.

41. Wang J, Boerma M, Fu Q, et al: Radiation responses in skin and connective tissues: effect on wound healing and surgical outcome. Hernia 10: 502-506, 2006.
42. Shamberger R: Effect of chemotherapy and radiotherapy on wound healing: experimental studies. Recent Results Cancer Res 98: 17-34, 1985

43. Gu Q, Wang D, Cui C, et al: Effects of radiation on wound healing. J Environ Pathol Toxicol Oncol 17: 117-123, 1998.

44. Grillo HC and Potsaid MS: Studies in wound healing. IV. Retardation of contraction by local $\mathrm{x}$-irradiation, and observations relating to the origin of fibroblasts in repair. Ann Surg 154: 741-750, 1961.

45. Tibbs MK: Wound healing following radiation therapy: a review. Radiother Oncol 42: 99-106, 1997.

46. Dobbs W: A statistical study of the effect of roenttgen rays on wound healing. AJR 41: 625-632, 1939.

47. Ormsby MV, Hilaris BS, Nori D, et al: Wound complications of adjuvant radiation therapy in patients with soft-tissue sarcomas. Ann Surg 210: 93-99, 1989.

48. Harris RP, Helfand M, Woolf SH, et al: Current methods of the US Preventive Services Task Force: a review of the process. Am J Prev Med 20: 21-35, 2001. 